OPEN ACCESS

Edited by:

James Lloyd,

Stellenbosch University, South Africa

Reviewed by:

Ganeshan Sivanandhan,

Chungnam National University,

South Korea

Taras P. Pasternak,

Albert Ludwig University of Freiburg,

Germany

*Correspondence:

Surekha Kundu

surekha_kundu@yahoo.com

Specialty section:

This article was submitted to

Plant Biotechnology,

a section of the journal

Frontiers in Plant Science

Received: 18 October 2016 Accepted: 10 March 2017

Published: 28 March 2017

Citation:

Chowdhury S, Basu A and Kundu S

(2017) Overexpression of a New Osmotin-Like Protein Gene (SindOLP)

Confers Tolerance against Biotic and Abiotic Stresses in Sesame.

Front. Plant Sci. 8:410.

doi: 10.3389/fp/s.2017.00410

\section{Overexpression of a New Osmotin-Like Protein Gene (SindOLP) Confers Tolerance against Biotic and Abiotic Stresses in Sesame}

\author{
Supriyo Chowdhury, Arpita Basu and Surekha Kundu* \\ Molecular and Applied Mycology and Plant Pathology Laboratory, Department of Botany, University of Calcutta, Kolkata, India
}

Osmotin-like proteins (OLPs), of PR-5 family, mediate defense against abiotic, and biotic stresses in plants. Overexpression in sesame of an OLP gene (SindOLP), enhanced tolerance against drought, salinity, oxidative stress, and the charcoal rot pathogen. SindOLP was expressed in all parts and localized to the cytosol. The transgenic plants recovered after prolonged drought and salinity stress, showing less electrolyte leakage, more water content, longer roots, and smaller stomatal aperture compared to control plants. There was an increase in osmolytes, ROS-scavenging enzymes, chlorophyll content, proline, secondary metabolites, and reduced lipid peroxidation in the transgenic sesame under multiple stresses. The OLP gene imparted increased tolerance through the increased expression of three genes coding for ROS scavenging enzymes and five defense-related marker genes functioning in the JAVET and SA pathways, namely Si-Apetala2, Si-Ethylene-responsive factor, Si-Defensin, Si-Chitinase, and Si-Thaumatin-like protein were monitored. The transgenic lines showed greater survival under different stresses compared to control through the integrated activation of multiple components of the defense signaling cascade. This is the first report of transgenic sesame and first of any study done on defense-related genes in sesame. This is also the first attempt at understanding the molecular mechanism underlying multi-stress tolerance imparted by an OLP.

Keywords: defense signaling, osmotin-like protein, charcoal rot, drought, salinity, over-expression, sesame, GFP

\section{INTRODUCTION}

Combinations of abiotic and biotic stresses, instead of individual ones, create a realistic threat to crop cultivation throughout the world. The concurrent presence of an abiotic stress either aggravates or impedes the effect of infection by a pathogen, thereby rendering a plant susceptible or tolerant against the combined stresses (Ramegowda and Senthil-Kumar, 2015). This is particularly important in case of the important oil/seed crop sesame which flower during the dry hot season when drought-induced reduction in cellular water-potential makes the plants more vulnerable to charcoal rot disease by Macrophomina phaseolina (Chowdhury et al., 2014a). Although, India is the largest producer of sesame in world producing 0.63 million tons/year, there is about $50 \%$ loss in yield due to the different biotic and abiotic stresses (Deepthi et al., 2014). 
Recent studies pointed out that under combined abiotic and biotic stresses, early defense signaling events in plants are likely operated by phytohormones but however the modulation of stress signal, crosstalk, and subsequent downstream events are later tailored for specific stress responses (Ramegowda and Senthil-Kumar, 2015). Stress signaling in plants in response to abiotic and biotic factors can induce separate and overlapping sets of genes, leading to the expression of distinct as well as common components (Zhu et al., 2014; Mellacheruvu et al., 2016). These separate pathways show nodal points where they converge and cross-talk to optimize the various defense responses (Xiao et al., 2013), resulting in shared stress mitigation strategy by combined morpho-physiological processes as well as by molecular responses (Pandey et al., 2015). Identification of cross talks between abiotic and biotic signaling pathways has been crucial for envisaging and strengthening our understanding of regulation of plants response against combined stresses. Genes such as those coding for Osmotins or Osmotin-like proteins that impart abiotic as well as biotic stress tolerance therefore present an opportunity to study the mechanism underlying tolerance to dual stresses.

Osmotin-like proteins (OLPs), that resemble Osmotin, a group of $24-26 \mathrm{kDa}$ proteins belonging to the PR5 group, was originally isolated from tobacco cells under osmotic stress (Choi et al., 2013). Under drought or salinity stresses, osmotin/osmotin like proteins (OLPs) maintains cellular osmolarity by compartmentalization of solutes or by structural and metabolic alterations. Recently we have cloned and characterized a new OLP gene from Solanum nigrum (SindOLP, BankIt 1588324 Solanum KC292261), which exhibited in-vitro antifungal activity against $M$. phaseolina (Chowdhury et al., 2015). As reported in previous studies, the synthesis and accumulation of OLPs depend on osmotic conditions, signifying its involvement in osmotic adaptations (Patade et al., 2013; Weber et al., 2014; Chowdhury et al., 2015). On the other hand a few reports have shown that osmotins and OLPs enhance tolerance to biotic stresses (Subramanyam et al., 2012; Vasavirama and Kirti, 2012; Choi et al., 2013), although the underlying biological mechanism remains unexplored. None of these studies showed that a single OLP gene can confer resistance to abiotic as well as biotic stresses in the same plant, leading to multi-stress resistance. Moreover, no prior studies investigated how the overexpression of an OLP gene affects the regulation of other defense-related genes in plants. The effect of OLP transgene on the regulation of marker genes functioning in the different signal transduction pathways leading to multiple stress tolerance therefore remains largely unknown.

Most of the studies on multi-stress resistance have been done on model plants. Studies on multi-stress resistance in other plants are few and even fewer studies explore the defense signaling mechanism(s) underlying multi-stress resistance in economically important plants (Zhu et al., 2014). In spite of the importance of sesame as an oil and seed crop, development of transgenic sesame was not feasible until recently due to the unavailability of a transformation protocol. The main obstacle was the severe recalcitrancy in-vitro that was overcome in our laboratory by establishment of a high frequency transformation protocol for sesame (Chowdhury et al., 2014b). Moreover, there is no study thus far about any gene regulation in sesame in response to any kind of stress. The recent availability of the sesame genome sequence (Zhang et al., 2013) is likely to change that scenario.

Here we overexpressed the new SindOLP in sesame not only to study its potentiality in conferring abiotic and biotic stress resistance but more importantly to get and insight into the mechanism by which the stress signaling takes place in these transgenic plants. The subsequent metabolic and molecular changes in response to biotic and abiotic stresses were observed in these transformed lines. The ability of the plants to recover after stress conditions and the expression of genes related to ROS scavenging system were studied. Five defense-related marker genes functioning in the JA/ET and SA signaling arms of defense response to multiple stresses were monitored in the transgenic plants to get an insight into the role of SindOLP in multi-stress tolerance. To the best of our knowledge, this is the first report of transgenic sesame. Moreover, this is also the first study of defense-related genes in sesame and the first attempt to analyze the molecular mechanism underlying enhancement of multistress tolerance by an OLP.

\section{MATERIALS AND METHODS \\ Cloning of Full Length SindOLP into Binary Vector}

Cloning of SindOLP from genomic DNA of S. nigrum L. in pBSKS (Stratagene) vector was done according to our earlier published report (Chowdhury et al., 2015). It was sub-cloned under CaMV35S promoter at the BamH1/Sal1 site of the plant binary vector pZPY112 (Figure 1A). The pZPY112::SindOLP was used to transform Agrobacterium tumefaciens LBA4404 strain by freeze-thaw method (Chen et al., 1994) and used for sesame transformation.

\section{Transformation of Sesame}

The pZPY112::SindOLP was used for transformation of sesame ( $S$. indicum cv.VRI-1) according to our earlier report (Chowdhury et al., 2014b). The transformants were regenerated on selection medium containing $50 \mathrm{mgL}^{-1}$ Kanamycin and 500 $\mathrm{mgL}^{-1}$ Cefotaxime. A total of 13 independent transformants were obtained by preliminary screening in Kanamycin (50 $\mathrm{mg} / \mathrm{L}$ ) and PCR screening using SindOLP primers (Figure 1B). Selected plantlets were transferred onto rooting medium (MS basal salts with $3 \%$ sucrose, $4.57 \mu \mathrm{M}$ IAA and $10 \mathrm{mgL}^{-1}$ Kanamycin). Transformants were sub-cultured three times onto fresh regeneration medium containing Kanamycin. After rooting, shoots were transferred into pots filled with soil-rite (soil substitute, Keltech, India). The primary transgenic lines $\left(\mathrm{T}_{0}\right)$ were self-pollinated and their seeds $\left(\mathrm{T}_{1}\right)$ were germinated in MS. Leaves from different T1 individuals from common parental T0 line were checked for kanamycin resistance and data were analyzed using Chi-square $\left(\chi^{2}\right)$ test as described earlier (Chowdhury et al., 2014b). 
A
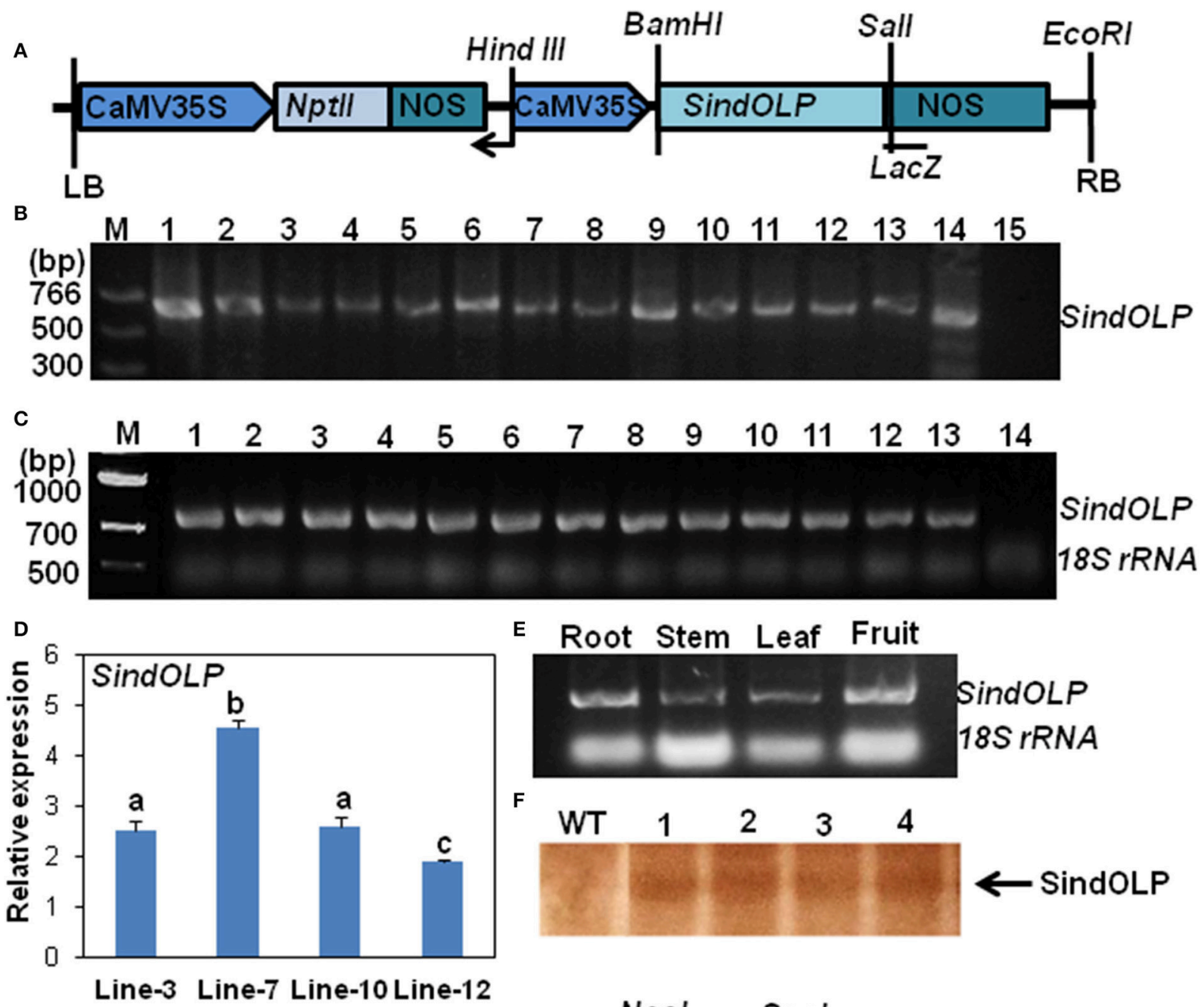

E Root Stem Leaf Fruit

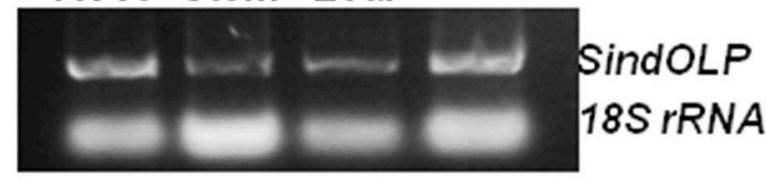

$\mathbf{F}$

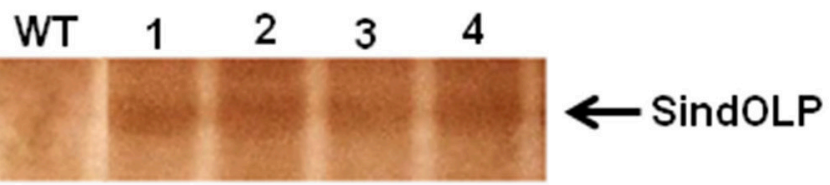

G
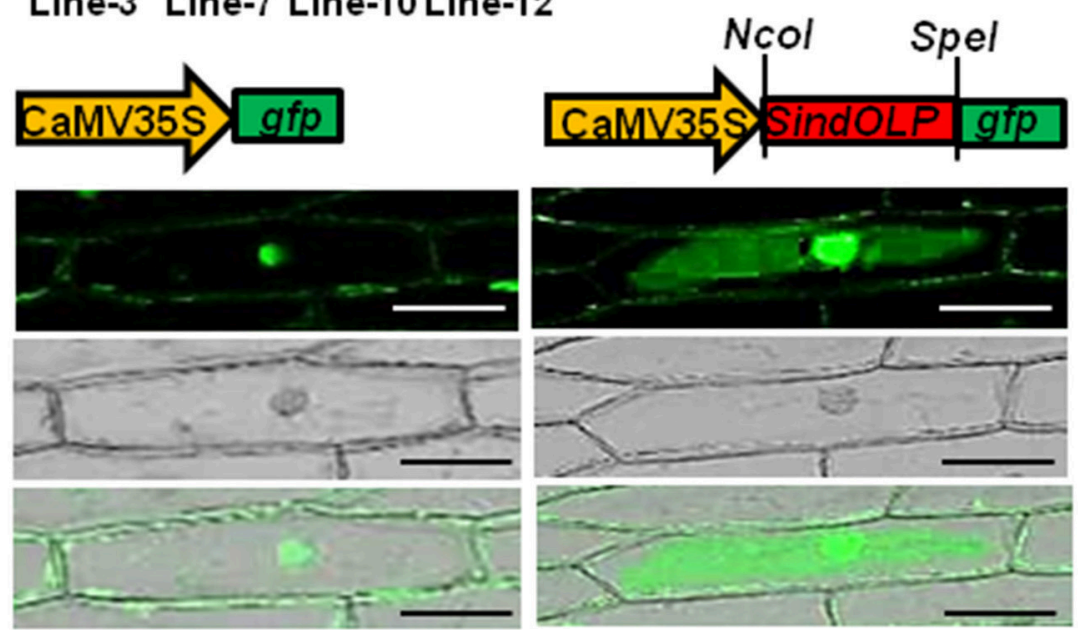

Fluorescence

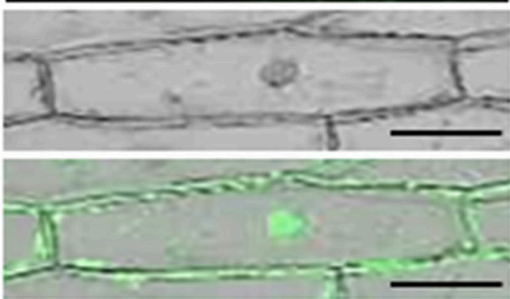

Bright field

FIGURE 1 | Molecular characterization of transformed sesame plants carrying SindOLP sequence. (A) Schematic representation of the T-DNA region of the construct pZPY112: SindOLP used for sesame transformation. NOS = nopaline synthesis terminator, Nptll = Neomycin phosphotransferase II (Kanamycin resistance) gene. (B) PCR analysis of genomic DNA from different transgenic lines using SindOLP specific primers. $M=$ molecular weight marker, $1-13=$ transgenic lines, $14=$ pZpy112: SindOLP plasmid (positive control), $15=$ WT sesame (negative control). (C) Expression of SindOLP in transgenic lines analyzed by semi-quantitative RT-PCR. 1-13 = transgenic lines, $14=$ non-transgenic plants, $18 S$ rRNA was kept as loading control. (D) Relative levels of SindOLP transcripts in four transgenic lines by q-RT-PCR. Bars represent mean \pm S.E.M of three independent experiments with three replicates. Different letters above bars represent significant difference at $P<0.05$. (E) Expression of SindOLP in different tissues of transgenic Line-7. (F) Immunoblot analysis of transgenic lines by anti-SindOLP antibody, $1=$ line-3, $2=$ line-7, $3=$ line-10, $4=$ line-12. (G) Subcellular localization of GFP-tagged SindOLP in onion epidermal cells (Bar $=200 \mu \mathrm{m})$. 


\section{Transformation of Macrophomina phaseolina with GFP Construct}

The charcoal rot pathogen $M$. phaseolina was transformed using binary vector pCambia 1302 (Supplementary Figure S8) using our previously published protocol (Basu et al., 2016).

\section{Detection of Copy Number of OLP of Transgenic Sesame Lines by q-RT-PCR}

Transformed lines were screened for SindOLP gene sequence by PCR. The full length ORF of $730 \mathrm{bp}$ SindOLP was amplified with primers SindOLP (f)-SindOLP(r) (Supplementary Table S4) using PCR conditions as published before (Chowdhury et al., 2015) and sequenced (Supplementary Figure S1B). Transgene copy number of SindOLP in the T0 and T1 lines were calculated by qRT-PCR using comparative $\mathrm{C}_{T}$ method. The sesame Oleosin gene (GenBank AF302807.1), a single copy gene in the sesame genome (Tai et al., 2002), was used as endogenous control for copy number calculation according to standard protocol (Weber et al., 2014; Chowdhury et al., 2014b).

\section{Protein Analysis by SDS-PAGE}

Total soluble protein was isolated from WT and transgenic plants according to Mandal et al. (2002). Fifty micrograms protein was loaded in each lane of a $12 \%$ SDS-PAGE and separated using a Mini-Protean II electrophoresis Cell (Bio-Rad laboratories, Hercules, CA).

\section{Detection of SindOLP in Transgenic Sesame Lines by Immunoblot}

His-tagged SindOLP, purified by Ni-NTA affinity chromatography (Chowdhury et al., 2015) was used to raise anti-SindOLP antisera in rabbit. For immunoblot analysis, $50 \mu \mathrm{g}$ of protein from WT and transgenic lines were separated by $12 \%$ SDS-PAGE and transferred onto PVDF membrane using Mini-Trans-Blot (Bio-Rad Laboratories, Hercules, CA). Immunoblotting was done using rabbit anti-SindOLP polyclonal antisera (1:200 dilution) and goat anti-rabbit horseradish peroxidase conjugated antibody (1:500 dilution; Genei, India).

\section{Subcellular Localization of SindOLp}

The complete ORF of SindOLP was amplified by PCR using primers NLS1-NLS2 (Supplementary Table S4, Supporting information) containing $\mathrm{NcoI}$ and SpeI site respectively and cloned into pCAMBIA 1302 vector to create pCAMBIA1302SindOLP-GFP (Figure 2C). The fusion construct and the control vector (pCAMBIA1302-GFP) were transformed into Agrobacterium LBA4404. Transformation of onion inner epidermal cells was done according to Huang et al. (2011). After 2 days on MS media the transformed cells were visualized under confocal laser scanning microscope (Olympus CLSM, Singapore Model no: 1X81) and images processed with Olympus Fluoview, FV1000.

\section{Evaluation of Response of Transgenic Sesame to Drought and Salinity Stress}

To assay the effect of stress on germination, surface-sterilized seeds from four homozygous transgenic lines (line-3, 7, 10, 12) and WT were germinated in MS-agar medium containing 0,100 , $200 \mathrm{mM} \mathrm{NaCl}$ (for salinity stress) and 0, 100, $200 \mathrm{mM}$ mannitol (for drought stress). For each treatment, 12 seeds from WT and different transgenic lines were placed in $1 / 2$ MS for 7 days at $28 \pm 1{ }^{\circ} \mathrm{C}, 16 / 8$ light: dark cycle and their germination frequencies were counted.

For stress on whole plants, WT and transgenic plants were grown in soil-rite in convirons at $70-75 \%$ relative humidity, $16 / 8$ light dark cycle, and $28 \pm 1^{\circ} \mathrm{C}$ for 10 weeks with regular watering on every alternate days. Drought stress was simulated by withholding water for 10 days. The plants were then allowed to recover for the next 2 days by watering. Plants were regarded as survivors if there were green, young leaves after the treatment. Survival rate was calculated as the ratio of number of surviving plants over the total number of treated plants. For salinity stress, plants were watered every day with $200 \mathrm{mM} \mathrm{NaCl}$ solution for 14 days. Each stress test was done three times.

\section{Estimation of Relative Water Content (RWC), Electrolyte Leakage (EL), Proline, Malonedialdehyde Content (Lipid Peroxidation), Phenolics, and Flavonoids}

RWC was measured according to $\mathrm{Hu}$ et al. (2013). Fresh weight (FW) of leaves was recorded followed by soaking the leaves for $4 \mathrm{~h}$ in distilled water at room temperature with constant light. The turgid weight (TW) was then recorded. Next the leaves were dried for $24 \mathrm{~h}$ at $80^{\circ} \mathrm{C}$ to obtain the dry weight (DW). RWC was calculated from the equation: RWC (\%) = $[(\mathrm{FW}-\mathrm{DW}) /(\mathrm{TW}-\mathrm{DW})] \times 100$.

Electrolyte leakage (EL) was measured according to $\mathrm{Hu}$ et al. (2013) with modification. Leaves were cut into strips and incubated in $10 \mathrm{~mL}$ distilled water at $28^{\circ} \mathrm{C}$ for $8 \mathrm{~h}$. Initial conductivity $(\mathrm{C} 1)$ was measured by a conductivity meter (Systronics, India) followed by boiling the sample in a water bath for $10 \mathrm{~min}$. The leaves were cooled to room temperature electrolyte conductivity (C2) was measured. EL was calculated according to the equation: $\mathrm{EL}(\%)=\mathrm{C} 1 / \mathrm{C} 2 \times 100$.

Proline and Malonedialdehyde content were estimated according to Chowdhury et al. (2011). Phenolic content and flavonoids were estimation according to Ray et al. (2015).

\section{Antioxidant Enzyme Activity}

Antioxidant enzymes viz. Ascobate peroxidase (APX; EC1.11.1.11) and Guaiacol peroxidase (GPX; EC1.11.1.7) were assayed according to Rai et al. (2013).

\section{Whole Plant and Detached Leaf Assay Using Macrophomina phaseolina}

For whole plant infection assay, infected soil treatment according to Kamalkannan et al. (2006) with modifications was utilized. For infected soil preparation, $100 \mathrm{ml}$ of microsclerotial suspension of M. phaseolina in sterile water $\left(1 \times 10^{6} \mathrm{microsclerotia} / \mathrm{ml}\right)$ was added to $100 \mathrm{~g}$ of autoclaved sand-maize medium (19:1 sand and ground maize grain) and allowed to grow for 2 weeks in dark at $28^{\circ} \mathrm{C}$. The inoculum was uniformly mixed with $2 \mathrm{~kg}$ sterile soil and incubated in dark for 7 days. Ten weeks old plants 


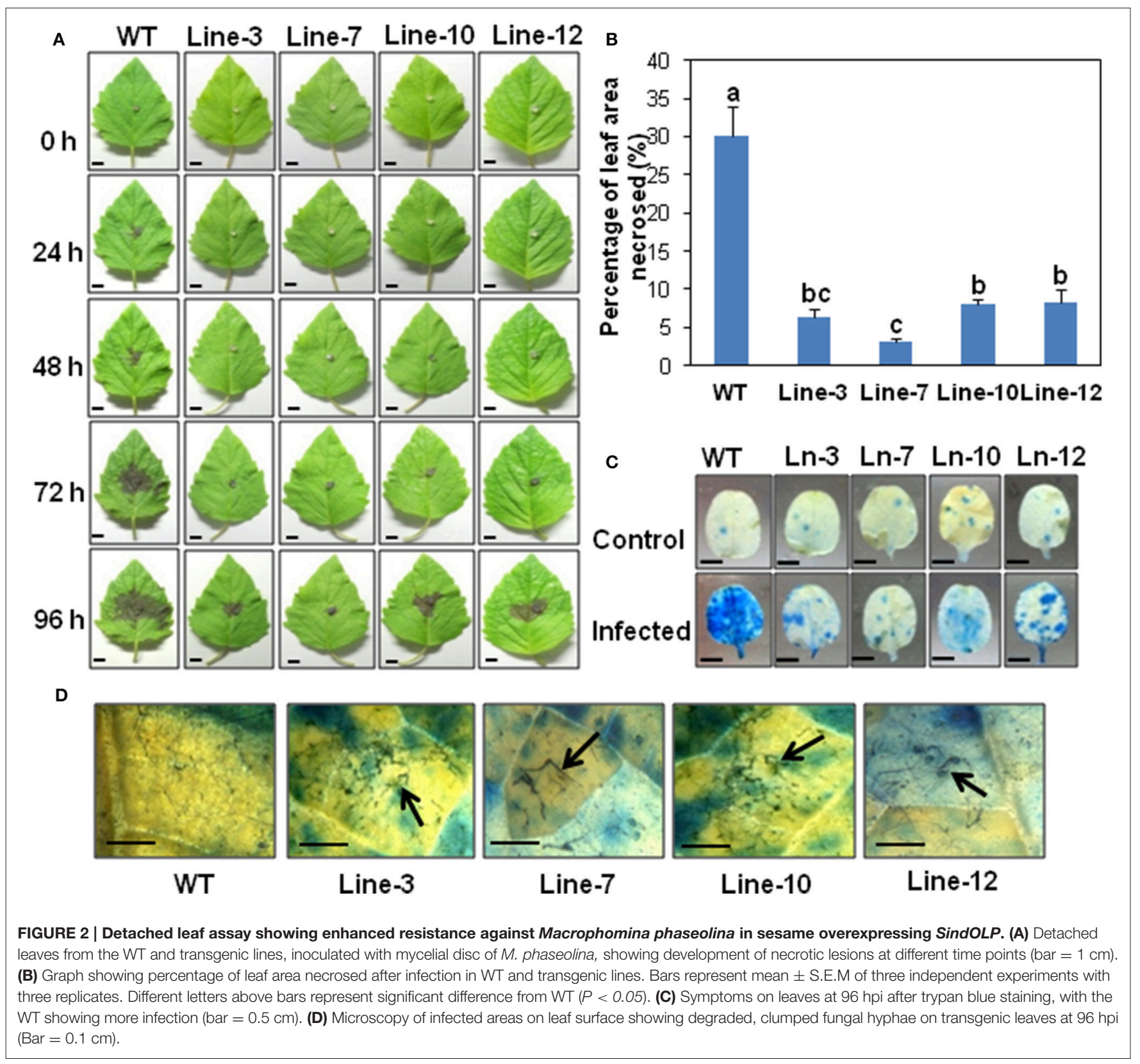

(WT and transgenic) were replanted in this infected soil, kept in convirons at $28^{\circ} \mathrm{C}, 16 / 8 \mathrm{~h}$ light dark cycle and watered at intervals of 3 days. After 30 days of planting, survival percentage was calculated.

For detached leaf assay, $3 \mathrm{~mm}$ discs containing microsclerotia of $M$. phaseolina were scooped out with a sterilized cork-borer and placed in the center of abaxial surface of detached leaves from 10 week old plants. Leaves were kept moist by placing them on wet filter paper inside convirons (temperature $28^{\circ} \mathrm{C}, 16 / 8 \mathrm{~h}$ of light dark cycle). The degree of infection was analyzed by the number of necrotic lesions at 24, 48, 72, 96 hpi and by trypan blue staining (Chowdhury et al., 2014a).

\section{Oxidative Stress Experiments and Measurement of Chlorophyll Content}

For assaying oxidative damage, healthy leaves from 3 week old seedlings (WT and transgenic lines) were floated on $5 \mathrm{ml}$ of $400 \mu \mathrm{M} \mathrm{H}_{2} \mathrm{O}_{2}$ for $24 \mathrm{~h}$. For the control set $5 \mathrm{ml}$ deionized water was used. After $24 \mathrm{~h}$ these were photographed and the extent of chloroplast damage was observed.

For measurement of chlorophyll content, chlorophyll was extracted from control and treated leaves by $80 \%$ acetone and O.D. was measured at 645 and $663 \mathrm{~nm}$ using a UV2300II spectrophotometer (Technocomp) according to Chowdhury et al. (2011). 


\section{Assay of $\mathrm{H}_{2} \mathrm{O}_{2}$ and Superoxide Anion Radicals by 3, 3'-Diaminobenzidine (DAB) and Nitro Blue Tetrazolium (NBT) Staining}

Three weeks old seedlings were subjected to stress treatments as follows: for salinity, sprayed with $200 \mathrm{mM} \mathrm{NaCl}$; for drought, sprayed with $200 \mathrm{mM}$ mannitol; for M. phaseolina infection, sprayed with microsclerotial suspension $\left(1 \times 10^{6}\right.$ microsclerotia/mL). After stress treatment for 3 days leaves were incubated in DAB $(2 \mathrm{mg} / \mathrm{mL})$ and NBT solution $(0.1 \mathrm{mg} / \mathrm{mL})$ for $24 \mathrm{~h}$ in dark at $28^{\circ} \mathrm{C}$ then soaked overnight in $95 \%$ ethanol to remove chlorophyll (Lu et al., 2013). For the control set, seedlings were sprayed with distilled water and stained as usual.

\section{Expression Analysis of Different Genes by Semi-Quantitative RT-PCR and q-RT-PCR}

For deciding on which sesame genes to study, five genes were selected (Supplementary Table S3) which are known to function in abiotic and biotic dual stress responses in other plants. These sequences were used to find corresponding genes in sesame from the sesame genome sequence (Zhang et al., 2013). The details of the work are given in Results Section. The list of genes is given in Supplementary Table S3.

Roots of 3 week old plants (lines-3, 7, 10, 12, and WT) were inoculated with M. phaseolina. Root were collected at different hours post inoculation (hpi) at the expected peak of expression for each genes viz. for SiAP2 at $36 \mathrm{hpi}, \operatorname{SiERF}$ at24 hpi, SiDef at $24 \mathrm{hpi}$, SiChi at $48 \mathrm{hpi}$, and SiTLP at $48 \mathrm{hpi}$. For RT-PCR and qRTPCR, RNA extraction and cDNA synthesis were done according to published protocol (Chowdhury et al., 2014b). All q-RT-PCR reactions were done following our protocol (Ray et al., 2015) (Applied Biosystems, USA).

Forward and reverse primers of the selected genes (Supplementary Table S4, Supporting information) were designed using Primer-3plus-software (http://www.bioinformatics.nl/cgi-bin/primer3plus/primer3plus. cgi) and IDT-oligo-analyzer (http://www.idtdna.com/analyzer/ Applications/OligoAnalyzer/). Amplified genes were sequenced to confirm their identity. Sesame 18S-rRNA and eIF4A were used as internal control for RT-PCR and q-RT-PCR respectively (Chowdhury et al., 2014b). The relative abundance of each gene was analyzed using the formula $2^{-\Delta \Delta \mathrm{Ct}}$. Three biological replicates were used to ensure the accuracy of results.

\section{Statistical Analyses}

Experimental data were subjected to analysis of variance (ANOVA) using software package used for statistical analysis (SPSS version 16, 2007). Significant difference of mean values was compared using Duncan's multiple range test (DMRT) at $P<0.05$. All the data are the mean \pm S.E.M of three independent experiments with three replicates.

\section{RESULTS}

\section{Molecular Characterization of Transgenic Sesame Lines}

Thirteen T0 lines having a single copy of SindOLP(detected by q-RT-PCR) were selected (Supplementary Figure S1,
Supplementary Table S1). After self-pollination, seeds from the $\mathrm{T}_{0}$ plants were collected and segregation of the transgene in $\mathrm{T} 1$ plants was analyzed by screening in MS medium containing Kanamycin $(50 \mathrm{mg} / \mathrm{L})$. Chi-square $\left(x^{2}\right)$ analysis showed 3:1 segregation for the NptII gene, indicating Mendelian segregation of single dominant gene in the selected $\mathrm{T}_{1}$ lines (Supplementary Table S2). T2 seeds coming from each $\mathrm{T}_{1}$ line were separately grouped. Twenty to Twenty-five seedlings of each group were again checked for Kanamycin resistance and for segregation of SindOLP sequence through PCR. The group of T2 seedlings that did not show segregation i.e., the group that were all Kanamycin resistant indicated that the mother T1 line was homozygous for the transgene. We selected four such T2 groups namely "line-3," "line-7," "line-10," and "line-12" for molecular and biochemical analysis and stress tolerance assays.

The level of transgene expression in T0 and T2 plants was monitored with "RT-PCR and q-RT-PCR." In the different T0 lines SindOLP expression is similar (Figure 1C). However, in $\mathrm{T}_{2}$ plants, there was considerable variation in SindOLP expression in the four selected lines (line-3, 7, 10, 12). Highest expression of SindOLP was seen in line-7 and lowest in line-12 (Figure 1D). Analysis of tissue specific expression of transgene in line-7 showed ubiquitous expression in different tissues viz. stem, leaf, root, and fruit (Figure 1E).

\section{Characterization of the Protein SindOLP in Transgenic Sesame Lines}

Total protein of the four T2 lines (line-3, 7, 10, 12) and WT were analyzed by SDS-PAGE. An extra protein band of $\sim 26$ $\mathrm{kDa}$, the expected size of recombinant SindOLP, was detected in protein samples of transgenic plants, which was not present in untransformed plants (Supplementary Figure S2A). The proteins were analyzed by western blot using antiserum against purified recombinant SindOLP. In western blot this $26 \mathrm{kDa}$ protein band was detected in transgeniclines, but not in the WT (Figure 1F and Supplementary Figure S2B).

\section{Subcellular Localization of SindOLP}

To observe the subcellular localization of SindOLP, SindOLP::GFP fusion construct, and GFP construct (control) driven by CaMV35S promoter were transiently expressed in the model system for protein localization, the onion epidermal cells (Figure 1G). Control GFP was found to localize in the nucleus and plasma membrane. The recombinant SindOLP::GFP was localized primarily in the cytosol, the periphery of the cells, the plasma membrane, at cell boundaries as well as in the nucleus (Figure 1G).

\section{Overexpression of OLP Confers of Fungal Resistance in Transgenic Sesame Smaller Necrotic Lesions in Transgenic Lines Compared to WT}

In detached leaf assays, at $96 \mathrm{hpi}$, symptoms on transgenic leaves consisted of restricted areas of hypersensitive necrotic zones, as opposed to WT leaves which showed extensive areas of necrosis (Figure 2A). The percentage of leaf area affected by necrosis in transgenic lines was significantly less compared to the WT (Figure 2B). Trypan blue staining 
confirmed less damage of leaf tissues in the transgenic lines compared to WT plants (Figure 2C). Stereo microscopy revealed the presence of damaged fungal hyphae on the leaf surface of transgenic sesame (Figure 2D), indicating the inhibitory role of SindOLP in restricting fungal growth, as studied earlier by in-vitro assays (Chowdhury et al., 2015).

\section{Whole Plant Bioassays Show Overexpression of SindOLP Leads to Enhanced Tolerance against M. phaseolina}

During whole plant infection assays, within 30 days after planting in infected soil, the WT plants were heavily infected showing typical symptoms of charcoal rot i.e., chlorosis and browning of leaves, wilting of aerial parts, stunted root growth, and dense accumulation of microsclerotia covering the lower stem giving it a charcoal black color. In contrast, the transgenic T2 lines showed less external symptoms (Figures 3A,B). Sections of infected roots and stems of WT and T2 lines revealed fungal colonization with Trypan blue staining and GFP fluorescence. In the WT plants, the roots were heavily colonized with fungal hyphae and in places the xylem vessels were clogged with microsclerotia (Figure 3C). The hyphal growth was found to be impeded in the root tissues of the transgenic plants (Figures 3C,D). Stem sections of WT plants showed signs of rot in the cortical tissue and the conducting vessels (Figure 3E), but no such colonization was seen in stem sections of transgenic plants. The T2 lines showed a higher plant survival (about 60-75\%) in comparison to the WT (about 15\%; Figure 3F).

\section{SindOLP Overexpressing Sesame Lines Showed Better Tolerance to $M$. phaseolina Infection Estimated by Biochemical Studies}

Under normal conditions, proline levels were similar in the leaf tissues of transgenic and WT plants. Although, the proline content increased after $M$. phaseolina infection in both, it was significantly higher in transgenic plants (480.09$553.01 \mu \mathrm{g} / \mathrm{g})$ compared to the WT $(365.69 \mu \mathrm{g} / \mathrm{g}$; Figure 4A). Amongst the transgenic lines, line-7 showed maximum increase of proline (Figure 4A). The MDA content, on the other hand, was significantly lower in the transgenic lines $(6.504-7.75 \mu \mathrm{mol} / \mathrm{g})$ than the WT plants $(12 \mu \mathrm{mol} / \mathrm{g})$ after infection (Figure 4B). In the transgenic lines, there were significant increase in total phenolics $(17.38-19.34 \mathrm{mg} / \mathrm{g}$ ) and flavonoids $(101.33-114.67 \mu \mathrm{g} / \mathrm{g})$ in comparison to the WT $(8.25 \mathrm{mg} / \mathrm{g}$ and $65.46 \mu \mathrm{g} / \mathrm{g}$, respectively) after infection (Figures 4C,D). Similarly, infection resulted in significant increase in activity of antioxidant scavengers such as GPX and cytosolic APX (total enzyme activity including both of its isoforms) in the transgenic lines compared to their untransformed counter parts (Figures 4E,F). There were no differences in biochemical parameters amongst WT and VC plants after M. phaseolina infection (Supplementary Figure S3).

\section{Sesame Plants Overexpressing SindOLP Showed Altered Expression of Marker Genes Functioning in the JA/ET and SA Pathways during $M$. phaseolina Infection}

To understand the mechanism behind the increased stress tolerance, the regulation of five defense-related marker genes functioning in the JA/ET and SA pathways were analyzed. The published sesame genome sequence (Zhang et al., 2013) was used to identify candidate genes that are known to function in other plants in the signal transduction pathways in response to abiotic as well as biotic stress, some of them possibly working as crosstalk factors. To check whether overexpression of SindOLP in sesame results in activation of phytohormone defense signaling, transcriptional profiles of the selected genes that function in the two pathways were studied. Supplementary Table S3 shows the list of selected genes and the acronyms we have assigned to them. Supplementary Table S4 shows the list of primers used. The PCR products were sequenced to confirm amplification of the defense gene sequences.

The expression of the five selected genes in the transgenic lines namely SiAP2, SiERF, SiDef, SiChi, SiTLP were analyzed by RT-PCR and qRT-PCR. The expressions of these five defenserelated genes were similar in $\mathrm{WT}$ and transgenic lines prior to M. phaseolina infection (Figure 5A). However, after infection with the fungus, expression of the genes was significantly upregulated in the transgenic lines (Figure 5A). qRT-PCR analyses revealed that there was two- to three-folds increase in the expression of the defense genes in the transgenic lines compared to the WT in response to fungal infection (Figures 5B-F). No significant differences in expression of defense related genes as well as external symptoms were found in WT and VC plants after inoculation with M. phaseolina (Supplementary Figure S4).

\section{Abiotic Stress Tolerance in the Transgenic Sesame Lines}

Transgenic Sesame Overexpressing SindOLP Showed Salinity and Drought Tolerance through Altered Physiological Parameters

The seeds of the transgenic lines were assayed for drought and salinity tolerance (Figures 6, 7). Under drought and salinity treatments, germination frequency of seeds was reduced in all seeds, but the transgenic seeds fared better than the WT (Figures 6A, 7A). In $100 \mathrm{mM}$ mannitol (drought stress), seed germination frequency in the WT was significantly lower $(16.62 \%)$ than that of the transgenic lines which ranged from 41.66 to $47.91 \%$. Similarly in $100 \mathrm{mM} \mathrm{NaCl}$ (salinity stress), transgenic lines had higher seed germination frequency (43.74$52.08 \%)$ in comparison to the WT (12.5\%). At the highest drought $(200 \mathrm{mM}$ mannitol) and salinity $(200 \mathrm{mM} \mathrm{NaCl})$ conditions, seed germination was completely blocked for WT plants. However, some seeds from the transgenic lines could tolerate this extreme stress. These had a germination frequency of $29.16-33.33 \%$ in drought stress (Figure 6B) and $18.7-27.08 \%$ in salinity stress (Figure 7B).

Similarly, there was a significant reduction in root- and shootlength in response to drought and salinity stress, in the case of 


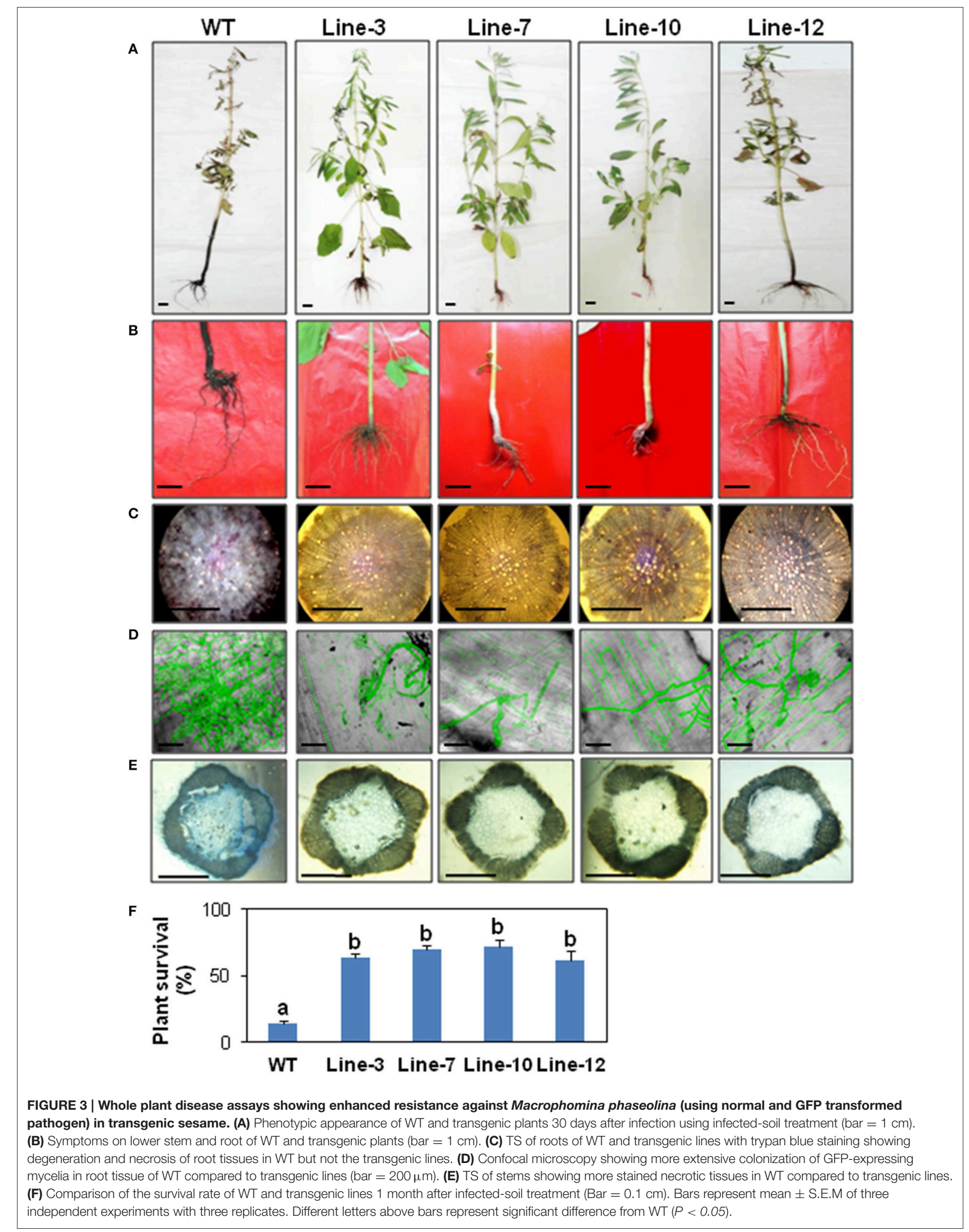



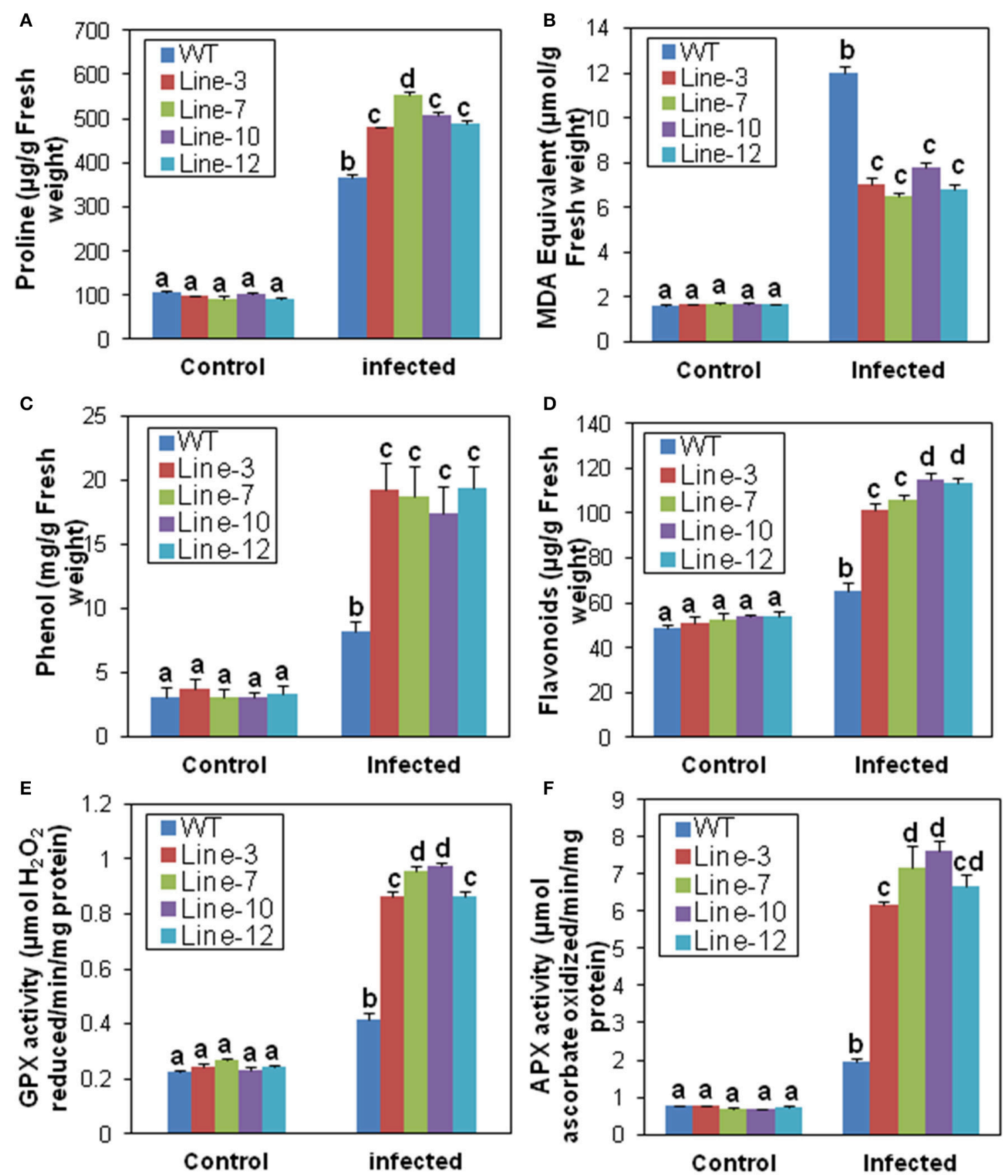

FIGURE 4 | Biochemical response of SindOLP overexpressing sesame plants against $\boldsymbol{M}$. phaseolina infection. Graphs showing comparison of different biochemical parameters in the WT and transgenic lines. (A) Proline (B) Lipid peroxidation (C) Phenol (D) Flavonoids (E) Guaiacol peroxidase (GPX) (F) Ascorbate peroxidase (APX) activity. Each bar represents mean \pm S.E.M of three independent experiments with three replicates. Different letters above bars represent significant difference from WT $(P<0.05)$.

WT plants compared to the transgenic lines at different stress levels (Figures 6C-E, 7C-E).

In survival experiments, most of the 10 weeks old WT plants after 10 days of drought treatment were irreversibly wilted but the transgenic plants were less affected (Figure 6F). Around $80 \%$ of transgenic plants recovered during re-watering compared to only $20 \%$ of the WT, indicating better sustenance of the transgenic lines under drought conditions (Figures 6G, H).

For survival assay under salinity stress, only $22 \%$ WT plants survived 14 days of salinity stress, whereas transgenic plants 


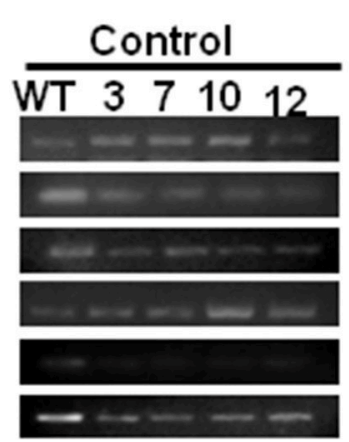

C

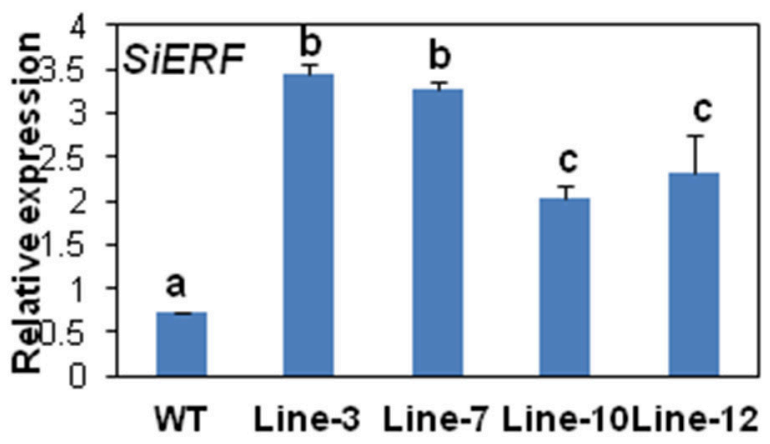

E

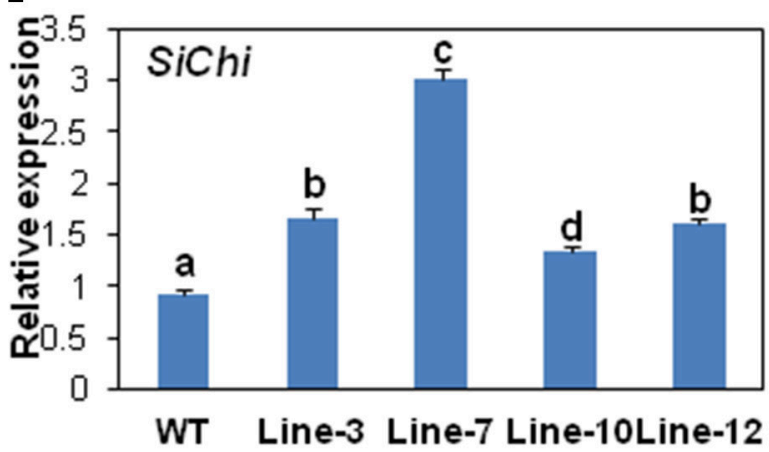

B

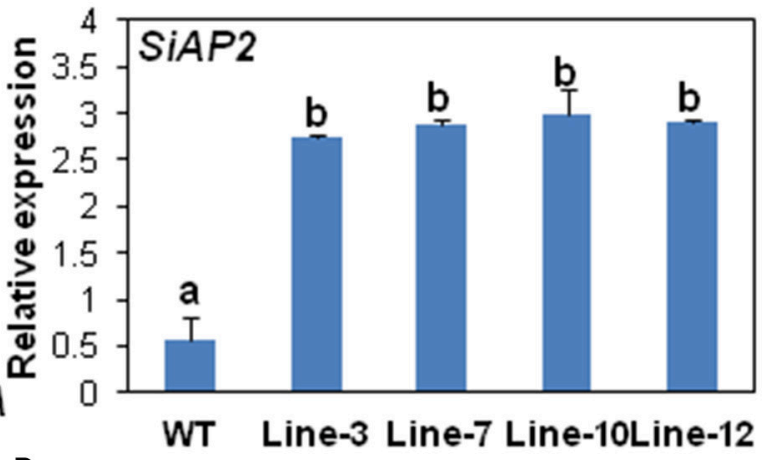

D

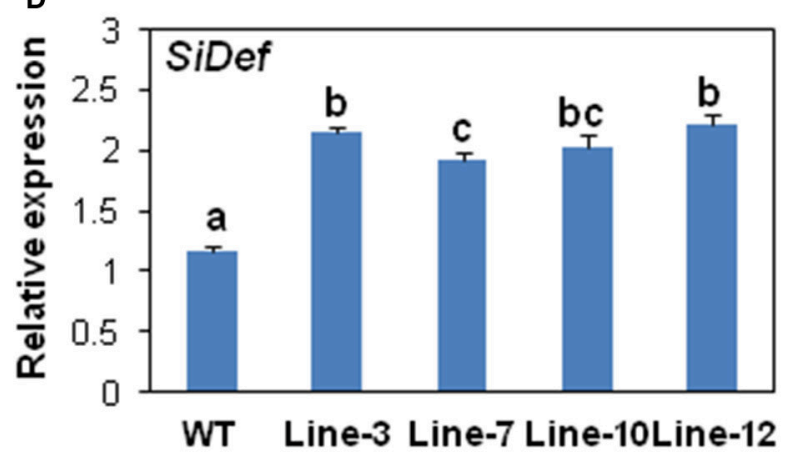

$\mathbf{F}$

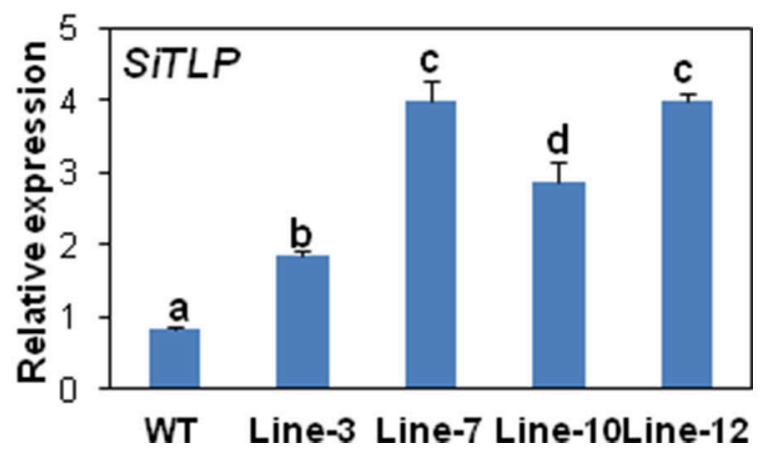

FIGURE 5 | SindOLP overexpression in sesame leads to up-regulation of defense genes acting in different arms of the signaling cascade upon M. phaseolina infection. (A) RT-PCR showing expression of five defense-related genes in WT and transgenic plants under control conditions and M. phaseolina infection. The selected marker genes acting in the JA/ET pathway are SiAP2 (Sesamum indicum EREBP/APETALA 2), SiERF(S. indicum ethylene-responsive transcription factor ERF071) and SiDef (S. indicum defensin) and those acting in the SA pathway are SiChi (S. indicum chitinase-like protein) and SiTLP (S. indicum thaumatin-like protein). (B-F) Graphs showing relative levels of the defense genes in WT and transgenic lines after infection with M. phaseolina; SiAP2 at 36 hpi, SiERF at $24 \mathrm{hpi}$, SiDef at $24 \mathrm{hpi}$, SiChi at $48 \mathrm{hpi}$, and SiTLP at $48 \mathrm{hpi}$. Bars represent mean \pm S.E.M of three independent experiments with three replicates. Different letters above bars represent significant difference from WT $(P<0.05)$.

showed significantly higher survival (68-84\%; Figure 7F). After 2 weeks of treatment, WT plants showed severe chlorosis and wilting but the transgenic plants sustained without visible signs of stress (Figure 7G).

Relative water content (RWC), an evaluation of plant water status, was higher in the transgenic lines than WT after 10 days drought stress. After re-watering, RWC increased in WT plants, but remained lower compared to the re-watered transgenic plants (Supplementary Figure S5A). Transgenic plants coped with water loss through well-developed root system with longer and thicker roots compared to WT plants (Supplementary Figures S5B,C). Electrolyte leakage, an important indicator of membrane damage, was significantly higher in WT plants than transgenic lines, suggesting less membrane damage during drought and salinity stress in the transgenic lines (Supplementary Figure S5D).

Since stomata often close in order to limit water loss by transpiration during abiotic stress like drought or salinity, stomatal apertures of the transgenic, and WT plants were compared before and after drought and salinity stress. The aperture of the open stomata was similar in the WT and 

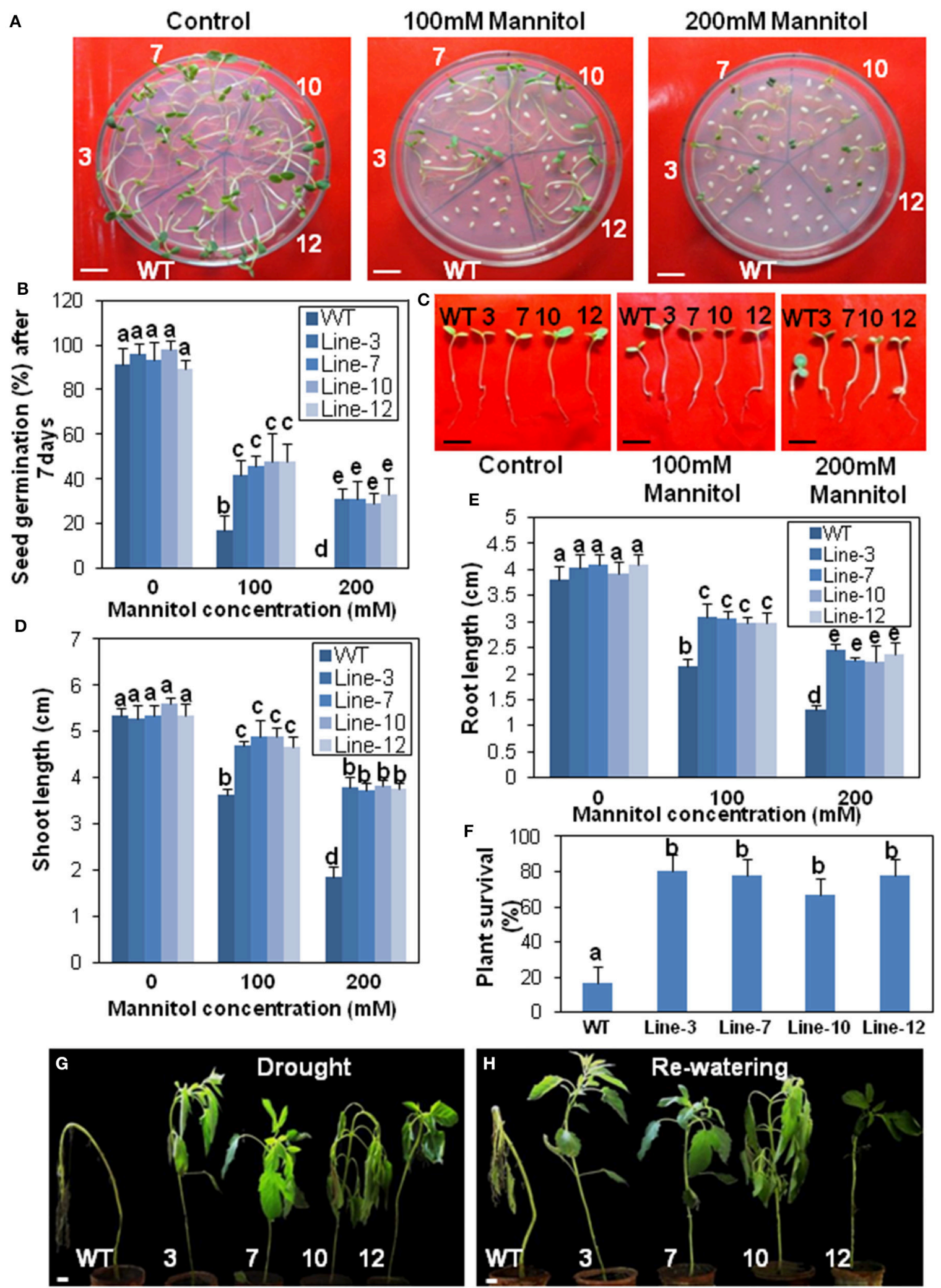

FIGURE 6 | Transgenic sesame overexpressing SindOLP has enhanced drought tolerance. (A) Germination of transgenic and WT seeds on MS medium with 0, 100, and $200 \mathrm{mM}$ mannitol (bar = $1 \mathrm{~cm}$ ). (B) Graph showing germination frequency of WT and transgenic lines under normal, 100 and $200 \mathrm{mM}$ mannitol treatment. 
FIGURE 6 | Continued

(C) Post germination seedling development in the WT and the transgenic lines on MS supplemented with 0, 100, and $200 \mathrm{mM}$ mannitol. The seeds sown on MS medium that showed radicle emergence after 3 days were transferred to MS medium containing different concentrations of mannitol. Photographs were taken 7 days after transfer $(\mathrm{bar}=1 \mathrm{~cm}) \mathbf{( D , E )}$ Primary shoot length and root length of seedlings 10 days after germination in different mannitol concentrations. $(\mathbf{F})$ Survival rates of 10 weeks old plants where drought conditions were simulated by withholding water for 10 days following 2 days of recovery by re-watering. Bars represent mean \pm S.E.M of three independent experiments with three replicates. Different letters above bars represent result significantly different from WT at $P<0.05$. (G,H) Representative photographs of 10 weeks old WT and transgeniclines grown under drought conditions for 10 days then watered for 2 days to allow for recovery (bar $=1 \mathrm{~cm})$.

transgenic plants before stress. However, during drought and salinity stress the SindOLP overexpressing plants exhibited a smaller stomatal aperture than the WT plants (Supplementary Figures S5E,F).

\section{Transgenic Sesame Over-Expressing OLP Showed Enhanced Drought and Salinity Tolerance through Altered Biochemical Parameters}

The proline accumulation in transgenic lines was significantly higher than WT plants after drought and salinity stress (Supplementary Figure S6A). The Malonedialdehyde (MDA) content of WT and the transgenic lines increased after drought and salinity stress in comparison to control. However, the MDA content of transgenic lines were significantly lower than that of WT (Supplementary Figure S6B) indicating less membrane injury in the transgenic plants. After stress, transgenic lines accumulated significantly higher phenolics and flavonoids than WT (Supplementary Figures S6C,D). Moreover, the transgenic lines showed significantly higher GPX and cytosolic APX activities than WT plants under stress (Supplementary Figures $\mathrm{S} 6 \mathrm{E}, \mathrm{F})$. There were no differences amongst WT and VC (vector control) plants after abiotic stresses (Supplementary Figure S3).

\section{SindOLP Overexpression Induces Oxidative Stress Tolerance in Sesame}

The leaves of WT plants changed from green to yellow after $\mathrm{H}_{2} \mathrm{O}_{2}$-induced oxidative stress for $24 \mathrm{~h}$, but the leaves from transgenic plants remained green (Figure 8A). The transgenic leaves had higher chlorophyll content $(65.93-70.1 \mathrm{mg} / \mathrm{g})$ than leaves from WT plants $(47.41 \mathrm{mg} / \mathrm{g})$ post oxidative stress (Figure 8B). There was no significant difference in chlorophyll content between the WT and VC plants before and after stress (Supplementary Figure S7).

\section{Transgenic Lines Showed Increased Transcript Levels of Three Enzymes Involved in ROS Signaling}

To understand the mechanism behind the increased oxidative stress tolerance, the expression of genes involved in ROS signaling viz. superoxide dismutase (SiSOD), cysteine protease inhibitor ( $\mathrm{SiCysPI)}$, glutathione-S-transferase ( $\mathrm{SiGST}$; Supplementary Tables S3, S4) was observed. Under control conditions, there was no difference in the expression levels of SiSOD, SiCysPI, and SiGST between the WT and the transgenic plants. After $400 \mathrm{mM} \mathrm{H}_{2} \mathrm{O}_{2}$ treatment for $24 \mathrm{~h}$, there was higher accumulation of SiCysPI, SiSOD, and SiGST transcripts in transgenic plants than WT (Figure 8C).

\section{Transgenic Plants Overexpressing SindOLP Showed Reduced ROS Accumulation}

Under normal conditions, the leaves of WT and transgenic lines showed no NBT staining. After stress treatment the leaves of plants overexpressing SindOLP exhibited less blue staining than WT plants, indicating less accumulation of $\mathrm{O}_{2}^{-}$(Figure 8D). Likewise, $\mathrm{H}_{2} \mathrm{O}_{2}$ contents of the WT and transgenic plants were similar under normal conditions. After infliction of biotic and abiotic stresses, the WT plants took a deeper DAB (3, $3^{\prime}$-Diaminobenzidine) stain compared to the transgenic lines indicating that the accumulation of $\mathrm{H}_{2} \mathrm{O}_{2}$ was considerably higher in the WT than the transgenic plants (Figure 8E).

\section{DISCUSSION}

Plants, unlike animals cannot move away from the sources of stress (Huey et al., 2002). At any given time, each of the different parts of a plant is exposed to several abiotic and biotic stresses. How plants adjust their responses to each of the stresses simultaneously and yet coordinately is an interesting area of research.

The overexpression of SindOLP, cloned in this laboratory (Chowdhury et al., 2015), in sesame presented a feasible approach to not only generating multi-stress tolerant crop lines, but also to provide us with the opportunity to study plant response to abiotic and biotic stresses. This study is the first report of transgenic sesame and also the first study involving gene regulation in sesame. Moreover, we get an insight into the molecular mechanism as to how OLPs enhance multi-stress tolerance, which hitherto has not been investigated.

The transgenic sesame lines overexpressing SindOLP showed resistance against the abiotic stresses of drought and salinity and the biotic stress caused by a fungal pathogen. The transgenic lines showed better seed germination, root/shoot length, RWC, survival rate, and sustenance under drought/salinity stress. At the cellular level, salinity and drought stresses is known to cause accumulation of MDA, proline, and secondary metabolites like flavonoids and phenolics (Tang et al., 2014). In this study lower MDA and lipid peroxidation, higher accumulation of proline, secondary metabolites, and improved antioxidant enzyme activities in the transgenic lines accounted for enhanced tolerance against drought, salinity, and pathogen. Less electrolyte leakage in transgenic lines during stress, indicate less membrane injury in the cells than WT plants. Subcellular localization experiments showed that the SindOLP::GFP fusion protein localized predominantly in the cytosol and plasma membrane which is consistent with previous reports about a pepper osmotin 

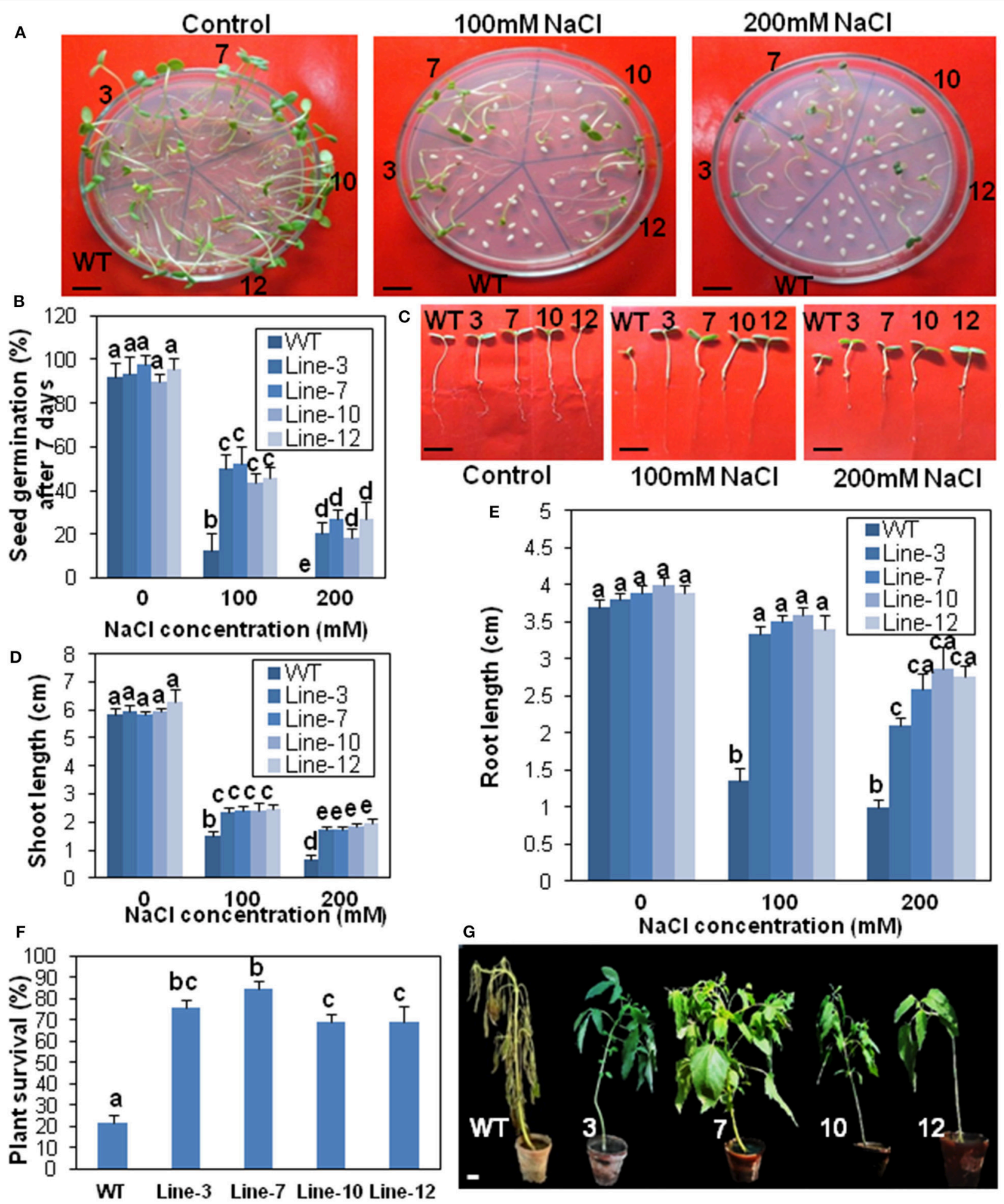

FIGURE 7 | Transgenic sesame overexpressing SindOLP has enhanced salinity tolerance. (A) Germination of transgenic and WT seeds on MS medium with 0, 100, and $200 \mathrm{mM} \mathrm{NaCl}$ (bar = 1 cm). (B) Graph showing germination frequency of WT and transgenic lines under normal, 100 and $200 \mathrm{mM} \mathrm{NaCl}$ treatment. (C) Post-germination seedling development of the WT and the transgenic lines on MS supplemented with 0, 100, and $200 \mathrm{mM}$ NaCl. The seeds sown on MS medium that showed radicle emergence after 3 days were transferred to MS medium containing different concentrations of NaCl. Photographs were taken 7 days after transfer $(\mathrm{bar}=1 \mathrm{~cm})$. (D,E) Primary shoot and root length of seedlings 10 days after germination in different concentrations of $\mathrm{NaCl}$. (F) Survival rates of 10 weeks old plants watered with $200 \mathrm{mM} \mathrm{NaCl}$ solution for 14 days. Bars represent mean \pm S.E.M of three independent experiments each with three replicates. Different letters above bars represent significant difference from WT at $P<0.05$. (G) Representative photographs of 10 weeks old WT and transgeniclines under salt stress for 14 days $(\mathrm{bar}=1 \mathrm{~cm})$. 


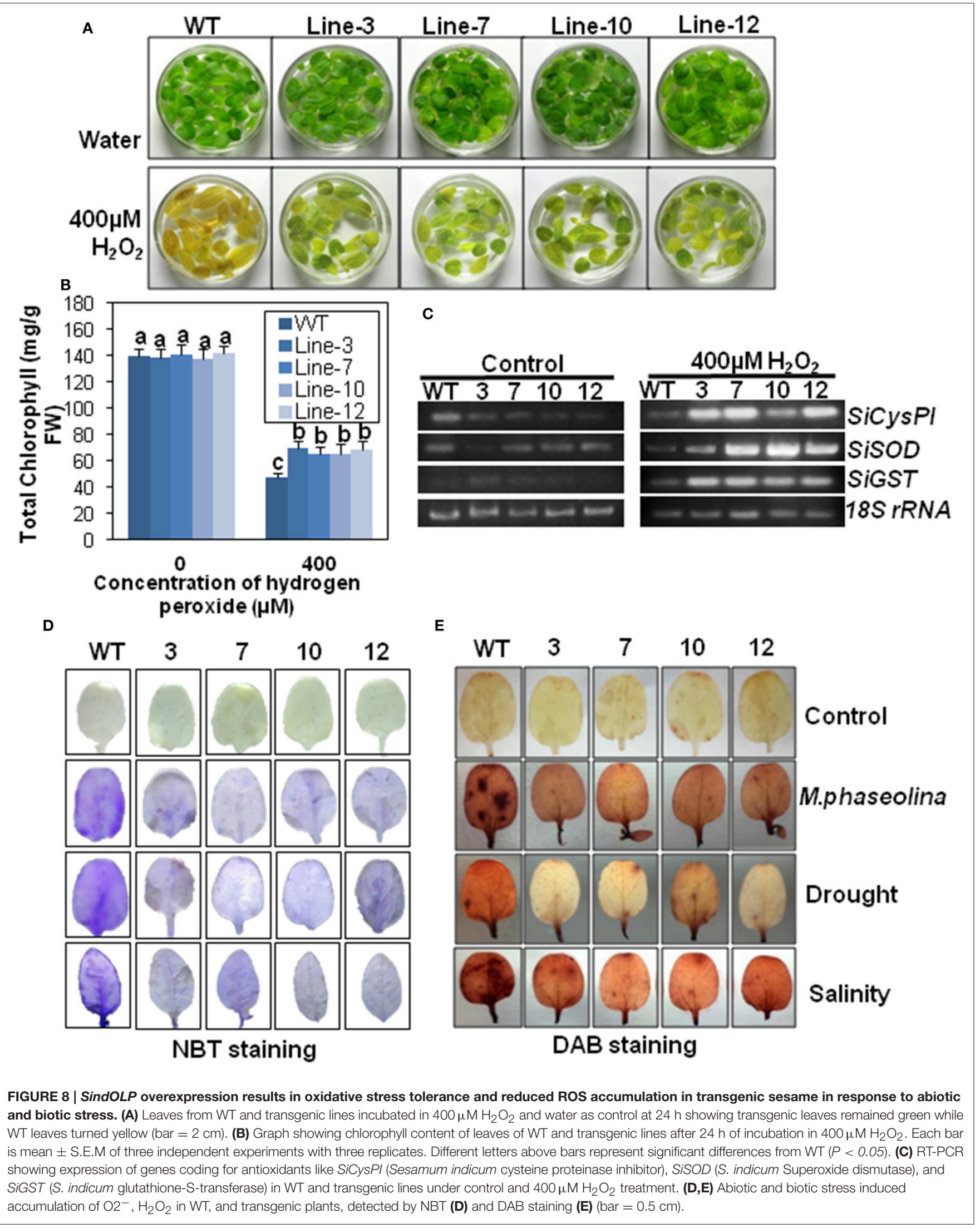


(Choi et al., 2013) and the thaumatin-like proteins (Kim et al., 2009).

Stomatal aperture is an important parameter in drought tolerance. The present study shows smaller stomatal apertures in the transgenic lines than WT leaves, presumably in an attempt to reduce water loss through transpiration and maintaining cellular osmolarity. Previous reports show that drought and salt tolerance is mediated through the control of stomatal aperture in rice (Huang et al., 2009) and maize (Cai et al., 2014).

Biotic and abiotic stresses commonly impinge on the redox status (Suzuki et al., 2014) and increase in activities of antioxidative enzymes is closely related to salt, drought, and pathogen resistance (Tamirisia et al., 2014). In this study the overexpression of SindOLP resulted in higher antioxidant activities in the transgenic lines. Although, incubation of leaves for DAB and NBT staining is stress itself, still less $\mathrm{H}_{2} \mathrm{O}_{2}$ and superoxide accumulation in transgenic lines compared to WT, under biotic/abiotic stress treatments speaks of less ROS accumulation as consequence of SindOLP overexpression. To elucidate the molecular mechanism behind the antioxidant role of SindOLP, we investigated the regulation of genes coding for ROS-scavenging enzymes in the transgenic lines. We found that $\operatorname{Sin} d O L P$ overexpression resulted in up-regulation of the ROS-scavenging enzyme genes viz. SiCysPI, SiSOD, and SiGST, during oxidative stress. Therefore, these results indicate that SindOLP takes part in the regulation of the ROS scavenging pathway.

Although, different in nature, salt, drought, and cold stresses are known to activate common sets of genes in plants (Tamirisia et al., 2014). Recent studies indicate that biotic stress also plays into the common network of defense signaling. The common nodal point from which the abiotic and biotic stress signaling pathways diverge, is around ROS production which in turn affect the two arms of phytohormone defense signaling viz. JA/ET and SA pathways (Kissoudis et al., 2014; Rejeb et al., 2014). In this study, to get an insight into the molecular mechanism behind the role of SindOLP in conferring resistance to abiotic and biotic stresses, we had selected marker genes that take part in the JA/ET and the SA signaling arms. The AP2, ERF, and Defensin are marker genes for the JA/ET pathway while Chi and $T L P$ are markers for SA signaling in plants. Expression analyses of these genes in sesame indicated that SindOLP-dependent activation of these defense genes plays a key role in enhanced

\section{REFERENCES}

Basu, A., Chowdhury, S., Ray Chaudhuri, T., and Kundu, S. (2016). Differential behavior of sheath blight pathogen Rhizoctonia solani in tolerant and susceptible and tolerant rice varieties before and during infection. Plant Pathol. 65, 1333-1346. doi: 10.1111/ppa.12502

Cai, G., Wang, G., Wang, L., Liu, Y., Pan, J., and Li, D. (2014). A maize mitogenactivated protein kinase kinase, $Z m M K K 1$, positively regulated the salt and drought tolerance in transgenic Arabidopsis. J. Plant Physiol. 171, 1003-1016. doi: 10.1016/j.jplph.2014.02.012

Chen, H., Nelson, R. S., and Sherwood, J. L. (1994). Enhanced recovery of transformants of Agrobacterium tumefaciens after freeze-thaw transformation and drug selection. Biotechniques 16, 664-668. abiotic/biotic stress tolerance in the transgenic plants, which is mediated through SA as well as JA/ET signaling pathways. Since OLPs are induced by SA and JA (Jami et al., 2007), the up-regulation of defense-related genes in transgenic sesame in response to fungal infection was due to the evocation of phytohormone defense signaling as a direct consequence of the constitutive overexpression of SindOLP. Although, the complex regulatory mechanism behind biotic/abiotic stress signaling involving SindOLP cannot be commented upon, this study indicate that it functions through integrated activation of several components of the stress signaling cascade.

On the whole it can be said that the overexpression of SindOLP in sesame led to improved drought, salinity, oxidative stress and disease tolerance through changes in some physiological, biochemical parameters and an integrated effect on the regulation of stress-responsive genes. Although, the complex regulatory mechanism involving SindOLP is not fully understood, our results give us an insight at the fundamental level, showing that the SindOLP gene has explicit functional role in abiotic/biotic stress signaling involving multiple components.

\section{AUTHOR CONTRIBUTIONS}

SK planned the project, drafted the experiments and acquired funding. $\mathrm{SC}$ and $\mathrm{AB}$ performed all the experiments and analyzed data. SC and SK wrote the manuscript. All authors read and approved the final version of the manuscript.

\section{ACKNOWLEDGMENTS}

This work was supported by DBT, India. Authors acknowledge partial financial support from University Grants Commission, Center of Advanced Studies Phase VII, Department of Botany, University of Calcutta. SC and $\mathrm{AB}$ received fellowships from UGC and CSIR, New Delhi respectively. The CLSM of DBTIPLS, Calcutta University and qPCR, Bose Institute, Calcutta were used.

\section{SUPPLEMENTARY MATERIAL}

The Supplementary Material for this article can be found online at: http://journal.frontiersin.org/article/10.3389/fpls.2017. 00410/full\#supplementary-material protein 1 (CaOSM1) is an essential component for defense response, cell death, and oxidative burst in plants. Planta 238, 1113-1124. doi: $10.1007 / \mathrm{s} 00425-013-1956-3$

Chowdhury, B., Chowdhury, S., and Biswas, A. K. (2011). Regulation of growth and metabolism in rice (Oryza sativa L.) by arsenic and its possible reversal by phosphate. J. Plant Interact. 6, 15-24. doi: 10.1080/17429140903487552

Chowdhury, S., Basu, A., and Kundu, S. (2014b). A new high-frequency Agrobacterium-mediated transformation technique for Sesamum indicum L. using de-embryonated cotyledon as explant. Protoplasma 251, 1175-1190. doi: 10.1007/s00709-014-0625-0

Chowdhury, S., Basu, A., and Kundu, S. (2015). Cloning, characterization and bacterial over-expression of an osmotin-Like protein gene from Solanum 
nigrum L. with antifungal activity against three necrotrophic fungi. Mol. Biotechnol. 57, 371-381. doi: 10.1007/s12033-014-9831-4

Chowdhury, S., Basu, A., Raychaudhuri, T., and Kundu, S. (2014a). In-vitro characterization of the behaviour of Macrophomina phaseolina (Tassi) Goid at the rhizosphere and during early infection of roots of resistant and susceptible varieties of sesame. Eur. J. Plant Pathol. 138, 361-375. doi: 10.1007/s10658-013-0335-Z

Deepthi, P., Shukla, C. S., Verma, K. P., and Reddy, S. S. (2014). Yield loss assessment and influence of temperature and relative humidity on charcoal rot development in sesame (Sesamum indicum L.). Bioscan 9, 193-195.

Hu, W., Huang, C., Deng, X., Zhou, S., Chen, L., Li, Y., et al. (2013). TaASR1, a transcription factor gene in wheat, confers drought stress tolerance in transgenic tobacco. Plant Cell. Environ. 36, 1449-1464. doi: 10.1111/pce.12074

Huang, X. S., Luo, T., Fu, X. Z., Fan, Q. J., and Liu, J. H. (2011). Cloning and molecular characterization of a mitogen-activated protein kinase gene from Poncirus trifoliata whose ectopic expression confers dehydration/drought tolerance in transgenic tobacco. J. Exp. Bot. 62, 5191-5206. doi: 10.1093/jxb/err229

Huang, X. Y., Chao, D. Y., Gao, J. P., Zhu, M. Z., Shi, M., and Lin, H. X. (2009). A previously unknown zinc finger protein, DST, regulates drought and salt tolerance in rice via stomatal aperture control. Genes Dev. 23, 1805-1817. doi: $10.1101 /$ gad.1812409

Huey, R. B., Carlson, M., Crozier, L., Frazier, M., Hamilton, H., Harley, C., et al. (2002). Plants versus animals: do they deal with stress in different ways? Integr. Comp. Biol. 42, 415-423. doi: 10.1093/icb/42.3.415

Jami, S. K., Anuradha, T. S., Guruprasad, L., and Kirti, P. B. (2007). Molecular, biochemical and structural characterization of Osmotin-like protein from black nightshade (Solanum nigrum). J. Plant Physiol. 164, 238-252. doi: 10.1016/j.jplph.2006.01.006

Kamalkannan, A., Mohan, L., Valluvaparidasan, V., Mareeswari, P., and Karuppiah, R. (2006). First report of Macrophomina root rot (Macrophomina phaseolina) on medicinal coleus (Coleus forskohlii) in India. Plant Dis. 55, 302. doi: 10.1111/j.1365-3059.2005.01302.x

Kim, B. G., Fukumoto, T., Tatano, S., Gomi, K., Ohtani, K., Tada, Y., et al. (2009). Molecular cloning and characterization of a thaumatin-like proteinencoding cDNA from rough lemon. Physiol. Mol. Plant Pathol. 74, 3-10. doi: 10.1016/j.pmpp.2009.07.001

Kissoudis, C., Wiel, C. V. D., Visser, R. G. F., and Linden, G. V. D. (2014). Enhancing crop resilience to combined abiotic and biotic stress through the dissection of physiological and molecular crosstalk. Front. Plant Sci. 5:207. doi: 10.3389/fpls.2014.00207

Lu, W., Chu, X., Li, Y., Wang, C., and Guo, X. (2013). Cotton GhMKK1 induces the tolerance of salt and drought stress, and mediates defense responses to pathogen infection in transgenic Nicotiana benthamiana. PLoS ONE 8:e68503. doi: 10.1371/journal.pone.0068503

Mandal, S., Kundu, P., Ray, B., and Mandal, R. K. (2002). Precursor of the inactive $2 S$ seed storage protein from the Indian mustard Brassica juncea is a novel trypsin inhibitor: characterization, post-translational processing studies and transgenic expression to develop insect resistant plants. J. Biol. Chem. 277, 37161-37168. doi: 10.1074/jbc.M205280200

Mellacheruvu, S., Tamirisia, S., Vudem, D. R., and Khareedu, V. R. (2016). Pigeon pea Hybrid-Proline-Rich Protein (CcHyPRP) confers biotic and abiotic stress tolerance in transgenic rice. Front. Plant Sci. 6:1167. doi: 10.3389/fpls.2015.01167

Pandey, P., Ramegowda, V., and Senthil-Kumar, M. (2015). Shared and unique responses of plants to multiple individual stresses and stress combinations: physiological and molecular mechanisms. Front. Plant Sci. 6:723. doi: 10.3389/fpls.2015.00723

Patade, V. Y., Khatri, D., Kumari, M., Grover, A., Gupta, S. M., and Ahmed, Z. (2013). Cold tolerance in osmotin transgenic tomato (Solanum lycopersicum L.) is associated with modulation in transcript abundance of stress responsive genes. Springerplus 2:117. doi: 10.1186/2193-1801-2-117
Rai, G. K., Rai, N. P., Rathaur, S., Kumar, S., and Singh, M. (2013). Expression of rd29A:: AtDREB1A/CBF3 in tomato alleviates drought induced oxidative stress by regulating key enzymatic and non-enzymatic antioxidants. Plant Physiol. Biochem. 69, 90-100. doi: 10.1016/j.plaphy.2013.05.002

Ramegowda, V., and Senthil-Kumar, M. (2015).The interactive effects of simultaneous biotic and abiotic stresses on plants: mechanistic understanding from drought and pathogen combination. J. Plant Physiol. 176, 47-54. doi: 10.1016/j.jplph.2014.11.008

Ray, S., Mondal, S., Chowdhury, S., and Kundu, S.,(2015). Differential responses of resistant and susceptible tomato varieties to inoculation with Alternaria solani. Physiol. Mol. Plant Pathol. 90, 78-88. doi: 10.1016/j.pmpp.2015. 04.002

Rejeb, I. B., Pastor, V., and Mauch-Mani, B. (2014). Plant responses to simultaneous biotic and abiotic stress: molecular mechanisms. Plants 3, 458-475. doi: 10.3390/plants3040458

Subramanyam, K., Arun, M., Mariashibu, T. S., Theboral, J., Rajesh, M., Singh, N. K., et al. (2012). Overexpression of tobacco Osmotin (Tbosm) in soybean conferred resistance to salinity stress and inhibit infections. Planta 236, 1909-1925. doi: 10.1007/s00425-012-1733-8

Suzuki, N., Rivero, R. M., Shulaev, V., Blumwald, E., and Mitler, R. (2014). Abiotic and biotic stress combinations. New Phytol. 203, 32-43. doi: 10.1111/nph.12797

Tai, S. S., Chen, M. C., Peng, C. C., and Tzen, Z. T. (2002). Gene family of oleosin isoforms and their structural stabilization in sesame seed oil bodies. Biosci. Biotechnol. Biochem. 66, 2146-2153. doi: 10.1271/bbb.66.2146

Tamirisia, S., Vudem, D. R., and Khareedu, V. R. (2014). Overexpression of pigeon pea stress-induced cold and drought regulatory gene (CcCDR) confers drought, salt, and cold tolerance in Arabidopsis. J. Exp. Bot. 65, 4769-4781. doi: $10.1093 /$ jxb/eru224

Tang, L., Cai, H., Zhai, H., Luo, X., Wang, Z., Cui, L., et al. (2014). Overexpression of Glycine soja WRKY20 enhances both drought and salt tolerance in transgenic alfalfa (Medicago sativa L.). Plant Cell Tissue Organ Cult. 118, 77-86. doi: 10.1007/s11240-014-0463-y

Vasavirama, K., and Kirti, P. B. (2012). Increased resistance to late leaf spot disease in transgenic peanut using a combination of PR genes. Funct. Integr. Genomics 12, 625-634. doi: 10.1007/s10142-012-0298-8

Weber, R. L. M., Wiebke-Strohm, B., Bredemeier, C., Margis-Pnheiro, M., Brito, G. G., Rechenmacher, C., et al. (2014). Expression of an osmotin-like protein from Solanum nigrum confers drought tolerance in transgenic soybean. BMC Plant Biol. 14:343. doi: 10.1186/s12870-014-0343-y

Xiao, J., Cheng, H., Li, X., Xiao, J., Xu, C., and Wang, S. (2013). Rice WRKY13 regulates cross talk between abiotic and biotic stress signaling pathways by selective binding to different cis-elements. Plant Physiol. 163, 1868-1882. doi: 10.1104/pp.113.226019

Zhang, H., Miao, H., Wang, L., Qu, L., Liu, H., Wang, Q., et al. (2013). Genome sequencing of important oilseed crop Sesamum indicum L. Genome Biol. 14:401. doi: 10.1186/gb-2013-14-1-401

Zhu, X., Qi, L., Liu, X., Cai, S., Xu, H., Huang, R., et al. (2014). The wheat Ethylene Response Factor transcription factor PATHOGEN-INDUCED ERF1 mediates host responses to both the necrotrophic pathogen Rhizoctonia cerealis and freezing stresses. Plant Physiol. 164, 1499-1514. doi: 10.1104/pp.113. 229575

Conflict of Interest Statement: The authors declare that the research was conducted in the absence of any commercial or financial relationships that could be construed as a potential conflict of interest.

Copyright (c) 2017 Chowdhury, Basu and Kundu. This is an open-access article distributed under the terms of the Creative Commons Attribution License (CC BY). The use, distribution or reproduction in other forums is permitted, provided the original author(s) or licensor are credited and that the original publication in this journal is cited, in accordance with accepted academic practice. No use, distribution or reproduction is permitted which does not comply with these terms. 